\title{
Evaluation and Correction of Ground-Based Microwave Radiometer Observations Based on NCEP-FNL Data
}

\author{
Qing Li1,2,3*, Ming Wei1,2,3, Zhenhui Wang1,2,3, Yanli Chu4, Lina Ma1,2,3 \\ ${ }^{1}$ Collaborative Innovation Center on Forecast and Evaluation of Meteorological Disasters, Nanjing University of Information \\ Science \& Technology, Nanjing, China \\ ${ }^{2}$ Key Laboratory for Aerosol-Cloud-Precipitation, Nanjing University of Information Science \& Technology, Nanjing, China \\ ${ }^{3}$ School of Atmospheric Physics, Nanjing University of Information Science \& Technology, Nanjing, China \\ ${ }^{4}$ Institute of Urban Meteorological Research, CMA, Beijing, China \\ Email: *liqingstu@126.com
}

How to cite this paper: Li, Q., Wei, M., Wang, Z.H., Chu, Y.L. and Ma, L.N. (2019) Evaluation and Correction of Ground-Based Microwave Radiometer Observations Based on NCEP-FNL Data. Atmospheric and Climate Sciences, 9, 229-242.

https://doi.org/10.4236/acs.2019.92016

Received: February 25, 2019

Accepted: March 29, 2019

Published: April 1, 2019

Copyright $\odot 2019$ by author(s) and Scientific Research Publishing Inc. This work is licensed under the Creative Commons Attribution International License (CC BY 4.0)

http://creativecommons.org/licenses/by/4.0/

\section{c) (i) Open Access}

\begin{abstract}
Consistency between the brightness temperatures observed with a ground-based microwave radiometer and the brightness temperatures computed by forward modeling is important in many different data applications. Using the National Centers for Environmental Prediction-Final Operational Global Analysis (NCEP-FNL) dataset as a reference, the brightness temperature was obtained through the radiation transfer model for forward calculation. The problem of segmented features in long time of observational data from ground-based microwave radiometers (the so-called "jumping problem") was identified. By analyzing the deviation and correlation between the observational bright temperature data and the forward calculated data under clear sky conditions, a revised scheme is proposed for the bright temperature observational data. Data obtained with a ground-based microwave radiometer in Beijing from January 1, 2010 to December 31, 2011 around the date of liquid nitrogen calibration show that the correlation between the observed brightness temperatures and the forward computed brightness temperatures is better after correction, especially at 28 and $30 \mathrm{GHz}$. The "jumping" problem in the observational data for the brightness temperature is eliminated after correction and the time continuity of the observational data and its consistency with the forward calculated data based on the NCEP-FNL dataset are improved. The proposed correction scheme can be used both for real-time data quality control and to improve the accuracy of historical datasets obtained with poorly calibrated microwave radiometers or radiometers working in polluted environments.
\end{abstract}




\section{Keywords}

Atmospheric Remote Sensing, Data Correction, Forward Model, Regression

\section{Introduction}

Ground-based microwave radiometers are commonly used for atmospheric observations [1] and can be operated continuously with a typical temporal resolution of $1 \mathrm{~s}$. They can be used to monitor the temperature and humidity profiles of the atmospheric boundary layer [2]-[9], and unique liquid water content profiles [10] [11] [12] [13] and to detect lightning [14]. The number of ground-based microwave radiometers in use in China for both research and quasi-operational observations has increased rapidly in recent years [15]-[20] and long time data have been obtained. However, due to changes in the observation position during the monitoring period (changing the environment of the instrument), the deposition of atmospheric pollutants on the radome (resulting in lower radome transmittance) and the half-yearly calibration with liquid nitrogen in accordance with the technical requirements of the system (resulting in a discontinuous calibration coefficient), these long time observational data show segmented features referred to as the "jumping problem".

The output from a microwave radiometer is the brightness temperature, which is defined as the electromagnetic energy received by the radiometer at a certain reference frequency. Either inversion processing or data assimilation must be performed to convert the brightness temperature data into information on the atmospheric temperature, humidity and liquid water content profiles based on the theoretical radiative transfer model [21] [22]. The brightness temperature observed by the microwave radiometer is inaccurate as a result of the hardware and calibration techniques used and can be solved from the point of view of the hardware. However, corrections to take account of the hardware used and calibration techniques cannot be applied to historical observational data from microwave radiometers and therefore inversion cannot be applied to the temperature and humidity profile. For these historical data, there is no possibility of improving the quality of the hardware to improve data accuracy. This paper discusses whether a data processing method can be used to correct historical observational data. The brightness temperatures observed using a ground-based microwave radiometer and the brightness temperatures simulated using a radiative transfer model must be consistent and reconcilable with each other.

Consistency analysis based on a comparison of the observed brightness temperatures with the forward calculated brightness temperatures has been successfully used in the quality control of observations from satellite-borne microwave radiometers. For example, Lu et al. [23] found that the frequencies at some of the microwave channels onboard the FY-3 satellite varied after launch based on a comparison of the observed brightness temperatures with the brightness temperatures calculated using the European Centre for Medium-Range Weather 
Forecasts model output as the input to the radiative transfer model. They therefore suggested a correction scheme to improve the application of the FY-3 data. Goldberg et al. [24] found that measurements from the AMSU instrument onboard the NOAA 15 and NOAA 16 satellites varied with the scan angle based on a comparison between the observed and simulated brightness temperatures. Weng et al. [25] showed that there was a clear asymmetry in the measured radiance along the scan measured by the AMSU instrument. This was most obvious in the window channels and was probably caused by either the misalignment of the polarizer or errors in the antenna pointing angle. Weng et al. [26] concluded that the antenna properties (e.g. the main beam efficiency and the spillover contributions from the side lobes) must be known before computing the brightness temperatures from the antenna temperatures measured by the Advanced Technology Microwave Sounder onboard the Suomi National Polar-orbiting Partnership satellite.

Systematic deviations between the observed brightness temperature $\left(T_{B M}\right)$ and the corresponding forward computed brightness temperature $\left(T_{B C}\right)$ also exist in ground-based microwave remote sensing [21], where the difference between the antenna temperature and the brightness temperature in the direction of the beam center is about $2 \mathrm{~K}$ for a $6^{\circ}$ beam width [27]. Meunier et al. [28] studied the effects of the characteristics of the radiometer (e.g. the antenna beam width and the receiver bandwidth) on simulated radiometer measurements based on theoretical simulations in which the beam width calculation is performed using a Gaussian quadrature and neglecting the effects of the side lobes. Tipping calibration is considered to be an important technique for the absolute calibration of ground-based microwave radiometers [27] [29] [30], but this is time consuming and is often not performed as often as would be useful. Wang Z. et al. [31] proposed verifying the working status of ground-based microwave radiometers in Nanjing and Wuhan by comparing $T_{B M}$ and $T_{B C}$ on a clear day. Li Qing et al. [32] tried to correct the systematic deviation to improve the consistency between the $T_{B M}$ values observed with a 22-channel radiometer in Beijing and the calculated $T_{B C}$ series for January 1, 2010 to December 31, 2011 when the radiometer was suspected to be malfunctioning. A liquid nitrogen calibration was performed on December 22, 2010. The results clearly show that the consistency between $T_{B M}$ and $T_{B C}$ can be improved for most of the 22 channels, but for some channels, especially channels $7(28 \mathrm{GHz})$ and $8(30 \mathrm{GHz})$, the improvement in consistency between $T_{B M}$ and $T_{B C}$ is negligible. Further study of this issue is required.

We used the analysis method for the quality control of bright temperature data from a satellite-borne microwave radiometer as a reference. We used NCEP-FNL [33] dataset as a reference because it has a high accuracy and good representativeness and time-space continuity. These data were used to obtain the brightness temperature $T_{B C}$ through the radiation transfer model for forward calculation [21] [34] [35]. We then identified the "jumping" problem in $T_{B M}$ by analyzing the deviation and correlation between the $T_{B M}$ data and the forward calculated $T_{B C}$ data under clear sky conditions and propose a correction scheme for 
$T_{B M}$. The verification was performed using the observational $T_{B M}$ dataset for the period January 1, 2010 to December 31, 2011.

\section{Theoretical Analysis and Estimation}

According to Westwater et al. [21], when the atmosphere is approximated as parallel planar and the scattering can be ignored, the brightness temperature for the thermal radiance vertically downward to the ground is expressed by the radiative transfer equation

$$
T_{B}(0)=T_{B}(\infty) \tau(0, \infty)+\int_{0}^{\infty} k_{a}(z) T(z) \tau(0, z) \mathrm{d} z
$$

where

$$
\tau(0, z)=\exp \left\{-\int_{0}^{z} k_{a}\left(z^{\prime}\right) \mathrm{d} z^{\prime}\right\}
$$

is the transmittance of the atmosphere from height $z$ to the antenna $(z=0)$, $\tau(0, \infty)$ is the transmittance of the whole atmosphere, $T_{B}(\infty)$ is the cosmic brightness temperature, which was assumed here to be $2.9 \mathrm{~K}[21], T(z)$ is the temperature profile and $k_{a}(z)$ is the absorption coefficient due to the presence of oxygen and water vapor in the atmosphere under a clear sky. $k_{a}(z)$ depends mainly on the pressure, temperature, humidity and wave frequency [36] [37]. The forward calculations according to Equation (1) are based on the radiative transfer model of Westwater et al. [21], including vertical discretization and humidity transformation; the absorption coefficient for atmospheric oxygen and water vapor follows Liebe et al. [37]. Data from either radiosondes or the NCEP-FNL Operational Model Global Tropospheric Analyses [33] can be used as the input temperature and humidity profiles in the forward model. The simulation scheme performed well in an earlier series of studies on the quality control and application of ground-based microwave radiometer data [31] [32] [35] [38] [39].

The power obtained by an antenna looking vertically upwards (the antenna temperature) is expressed as [40]:

$$
T_{A}=\int_{4 \pi} T_{B}(\theta, \phi) F(\theta, \phi) \mathrm{d} \Omega / \int_{4 \pi} F(\theta, \phi) \mathrm{d} \Omega
$$

where $\theta$ and $\phi$ are the zenith and azimuth angles of the incident radiance toward the antenna, $\mathrm{d} \Omega=\sin \theta \mathrm{d} \theta \mathrm{d} \phi, F(\theta, \phi)$ is the radiation pattern of the antenna, $T_{B}(\theta, \phi)$ is the brightness temperature incident on the antenna from the direction $(\theta, \phi)$, of which $T_{B}\left(\theta=0^{\circ}, \phi\right)$ is from zenith and is equal to $T_{B}(0)$ defined by Equation (1).

In most situations for a parabolic antenna, $F(\theta, \phi)$ is approximated to 1 in the main beam and to 0 elsewhere, so $T_{A}=T_{B}(0)$ and Equation (1) can be used directly to simulate the observations from the antenna.

In real situations, the antenna side lobes are weak, but rarely 0 (Figure 1(a)). In addition, pollutants deposited on the radiometer radome result in a small but significant loss, which leads to emissions visible via the antenna main lobe that 


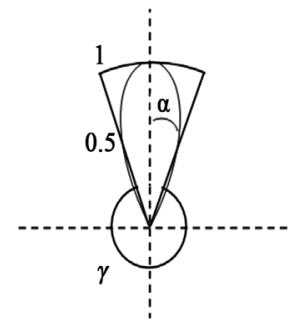

(a)

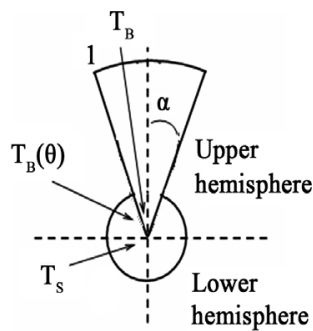

(b)

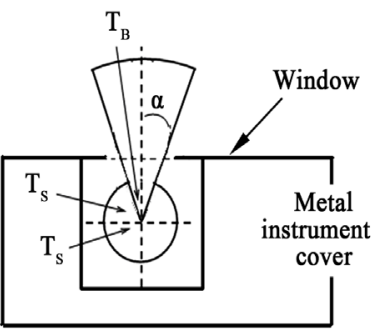

(c)

Figure 1. Schematic diagrams showing (a) the equivalence of the antenna radiation pattern; (b) the incident radiance and (c) the influence of the radome.

can correlated with the ambient temperature. Therefore, in order to predict the contamination of the atmospheric radiance measurement from the surroundings with an easy-to-understand formula, we assume that: the values of both $F(\theta, \phi)$ and $T_{B}(\theta, \phi)$ in the upper hemisphere are isotropic in the azimuth direction; $T_{B}(\theta)$ in the upward-looking main beam is identical to $T_{B}(0)$ and is representative of atmospheric radiance; and $T_{B}(\theta)$ in the lower hemisphere is equivalent to $T_{S}$ and is representative of the radiance from the surroundings (Figure 1(b)). Equation (3) then becomes

$$
\begin{aligned}
T_{A} & =\frac{2 \pi\left\{\int_{0}^{\alpha} T_{B}(0) F(\theta)+\int_{\alpha}^{\pi / 2} T_{B}(\theta) F(\theta)+\int_{\pi / 2}^{\pi} T_{S} F(\theta)\right\} \sin \theta \mathrm{d} \theta}{2 \pi \int_{0}^{\pi} F(\theta) \sin \theta d \theta} \\
& =T_{B}(0) \gamma_{1}+T_{B}(\bar{\theta}) \gamma_{2}+T_{S} \gamma_{3}
\end{aligned}
$$

where $\gamma_{1}, \gamma_{2}$ and $\gamma_{3}$ are parameters determined by the radiation pattern of the antenna and $T_{B}(\bar{\theta})$ is defined according to the integral mean value theorem.

Because the antenna reflector is covered by a metal cover containing a foam dielectric radome (Figure 1(c)), the second term in Equation (4) can be approximated using the contributions from both $T_{B}(0)$ and $T_{S}$ as the proportion $\beta$ and hence Equation (4) becomes

$$
T_{A}=T_{B}\left(\gamma_{1}+\beta\right)+T_{S}\left(\gamma_{3}+1-\beta\right)
$$

where $T_{B}=T_{B}(0)$ and $\beta$ is the proportional parameter and depends on the performance of both the antenna and the radome. A properly maintained or new radome can be taken as a perfect microwave window, but deposits on the radiometer radome, especially in areas with high levels of pollution, will increase the opacity of the radome and the value of $\beta$ will decrease.

According to Equation (5), the observations of atmospheric radiance are influenced by the surroundings and must be corrected. Because the contribution from $T_{S}$ can be mistaken as an increment in $T_{B}$, the correction to $T_{B}$ to take account of the surrounding environment according to Equation (5) is

$$
\delta T_{B}=-T_{S}\left(\gamma_{3}+1-\beta\right) /\left(\gamma_{1}+\beta\right)
$$

The effect of the surrounding environment on the antenna is complex, but, according to Westwater et al. [21], $T_{S}$ mainly depends on the temperature of the 
surroundings:

$$
T_{S} \approx \varepsilon_{*} T_{g}
$$

where $T_{g}$ and $\varepsilon$ are the surrounding temperature and emissivity, respectively.

\section{Verification of the Correction for the Surroundings}

To obtain $\delta T_{B}$, the amount of correction required to the value of $T_{B M}$ measured by the radiometer, it is assumed that the surrounding temperature is dominant and the emissivity is combined with $\left(\gamma_{3}+1-\beta\right) /\left(\gamma_{1}+\beta\right)$ in Equation (6) to give

$$
\delta T_{B}=c * T_{g}
$$

where $c$ is a coefficient for the correction to the surroundings.

The correction for a systematic deviation caused by the difference between the atmospheric profiles over the radiometer and the atmospheric profiles used as the input in the forward calculation for $T_{B C}$ based on Equation (1) is expressed as

$$
T_{\text {BO }}=a * T_{B M}+b
$$

where $a$ and $b$ are coefficients and $T_{B O}$ is the result obtained from the correction [32]. The combination of the correction for systematic deviations and the correction for the surroundings leads to a bivariate linear regression model

$$
T_{B O}=a * T_{B M}+b+c * T_{g}
$$

Considering that the forward calculated result $\left(T_{B C}\right)$, based on a radiative transfer equation such as Equation (1), is mostly used as a reference in data assimilation and profile inversion, let the residual sum of square $Q$ be expressed as

$$
Q=\sum\left(T_{B O}-T_{B C}\right)^{2}=\min
$$

i.e.,

$$
\begin{aligned}
& \frac{\partial Q}{\partial a}=2 \sum\left(a * T_{B M}+b+c * T_{g}-T_{B C}\right) * T_{B M}=0 \\
& \frac{\partial Q}{\partial b}=2 \sum\left(a * T_{B M}+b+c * T_{g}-T_{B C}\right)=0 \\
& \frac{\partial Q}{\partial c}=2 \sum\left(a * T_{B M}+b+c * T_{g}-T_{B C}\right) * \Delta T_{g}=0
\end{aligned}
$$

to obtain the optimum estimation for the coefficients $a, b$ and $c$ in Equation (9) from a sample of data.

The data sample used by Li Q. et al. [32] is used here for verification. The data sample is a radiometer with 35 channels, of which 22 are used for observations of the operational brightness temperature. The central frequencies for channels 1 - 8 are $22-30 \mathrm{GHz}$ in the $K$ band and the central frequencies for channels 9 - 22 are $51-59 \mathrm{GHz}$ in the $\mathrm{V}$ band (Table 1 ). The radiometer has its own thermometer for measuring the surface air temperature, which is taken as $T_{g}$ in the calculations. The observations were collected at 0800 and 2000 Beijing Time from January 1, 2010 to December 31, 2011. A total of 603 observations were made under clear skies. Clear sky conditions are defined as a relative humidity of 
Table 1. Bias $(K)$ and standard deviation (std, $K$ ) of the difference between the measured and calculated brightness temperatures and their correlation coefficient before and after correction.

\begin{tabular}{|c|c|c|c|c|c|c|c|}
\hline $\begin{array}{c}\text { Channel } \\
\text { index }\end{array}$ & $\begin{array}{c}\text { Frequency } \\
(\mathrm{GHz})\end{array}$ & $\begin{array}{l}\text { Bias before } \\
\text { correction }\end{array}$ & $\begin{array}{l}\text { Standard } \\
\text { deviation } \\
\text { before } \\
\text { correction }\end{array}$ & $\begin{array}{l}\text { Bias after } \\
\text { correction }\end{array}$ & $\begin{array}{l}\text { Std. after } \\
\text { correction }\end{array}$ & $\begin{array}{l}\text { Correlation } \\
\text { before } \\
\text { correction }\end{array}$ & $\begin{array}{c}\text { Correlation } \\
\text { after } \\
\text { correction }\end{array}$ \\
\hline 1 & 22.234 & 3.98 & 6.48 & 0.00 & 3.01 & 0.9313 & 0.9855 \\
\hline 2 & 22.500 & 3.31 & 6.88 & 0.00 & 3.11 & 0.9233 & 0.9849 \\
\hline 3 & 23.034 & 3.12 & 8.78 & 0.00 & 3.43 & 0.8587 & 0.9796 \\
\hline 4 & 23.834 & 3.43 & 8.39 & 0.00 & 2.86 & 0.8263 & 0.9803 \\
\hline 5 & 25.000 & 2.21 & 7.07 & 0.00 & 2.75 & 0.7738 & 0.9675 \\
\hline 6 & 26.234 & 2.03 & 5.97 & 0.00 & 2.70 & 0.7223 & 0.9474 \\
\hline 7 & 28.000 & 3.84 & 7.71 & 0.00 & 2.51 & 0.3909 & 0.9256 \\
\hline 8 & 30.000 & 4.54 & 10.15 & 0.00 & 2.73 & 0.1284 & 0.8814 \\
\hline 9 & 51.248 & -2.05 & 2.84 & 0.00 & 1.50 & 0.9078 & 0.9695 \\
\hline 10 & 51.760 & -1.29 & 3.41 & 0.00 & 1.41 & 0.8738 & 0.9752 \\
\hline 11 & 52.280 & -4.20 & 2.67 & 0.00 & 1.36 & 0.9506 & 0.9807 \\
\hline 12 & 52.804 & -4.50 & 2.60 & 0.00 & 1.20 & 0.9639 & 0.9883 \\
\hline 13 & 53.336 & -3.72 & 2.46 & 0.00 & 0.91 & 0.9761 & 0.9948 \\
\hline 14 & 53.848 & -1.60 & 1.92 & 0.00 & 0.65 & 0.9868 & 0.9978 \\
\hline 15 & 54.400 & -0.19 & 1.44 & 0.00 & 0.66 & 0.9923 & 0.9980 \\
\hline 16 & 54.940 & 0.18 & 1.20 & 0.01 & 0.69 & 0.9946 & 0.9980 \\
\hline 17 & 55.500 & 0.43 & 1.43 & 0.00 & 1.03 & 0.9921 & 0.9958 \\
\hline 18 & 56.020 & 0.40 & 1.32 & 0.00 & 0.92 & 0.9934 & 0.9968 \\
\hline 19 & 56.660 & 0.39 & 1.40 & 0.00 & 0.98 & 0.9928 & 0.9964 \\
\hline 20 & 57.288 & 0.44 & 1.50 & 0.00 & 1.08 & 0.9916 & 0.9956 \\
\hline 21 & 57.964 & 0.39 & 1.54 & 0.00 & 1.11 & 0.9912 & 0.9954 \\
\hline 22 & 58.800 & 0.38 & 1.64 & 0.00 & 1.16 & 0.9900 & 0.9950 \\
\hline
\end{tabular}

$<85 \%$ in the troposphere [34]. Only the data for clear sky days were used in the following statistical analysis to avoid the uncertain influence of clouds on the estimation of the coefficients. However, the results are applicable to all weather conditions because the coefficients account for both the systematic deviations and contamination from the surroundings. Additional corrections may be necessary for the effects of clouds.

1) Verification with time series and correlation analysis

Figure 2 shows the time series of $T_{B M}$ in the sample and the corresponding simulated values of $T_{B C}$ for four typical channels at 22.234, 28.000, 53.336 and $55.500 \mathrm{GHz}$. The gap between $T_{B M}$ and $T_{B C}$ includes the error in the radiative transmission mode and the space-time difference between radiometer data and the lattice data. This type of deviation is fairly limited. There are two jumps in the time series, one associated with the liquid nitrogen calibration at the 294th 


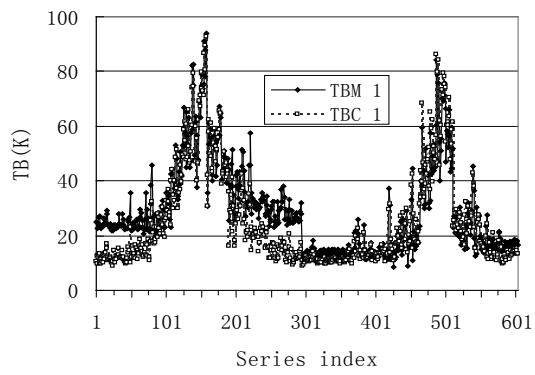

(a)

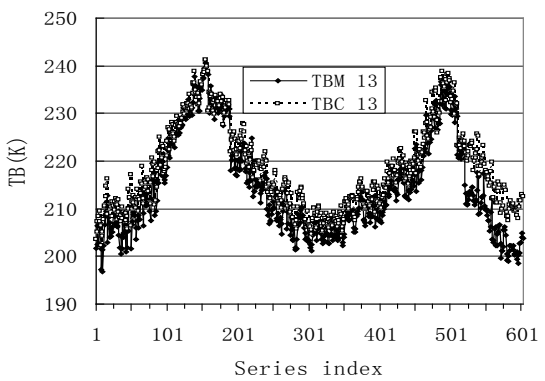

(c)

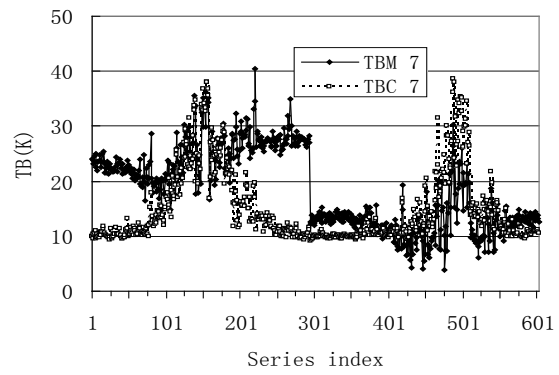

(b)

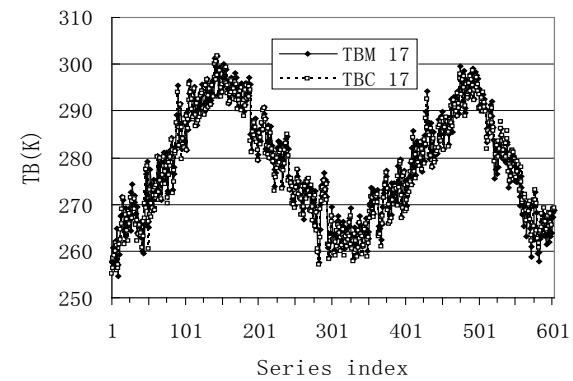

(d)

Figure 2. Comparison of the time series of the observed clear sky brightness temperatures $\left(T_{B M}\right)$ with the simulated temperatures $\left(T_{B C}\right)$ for four typical channels during the period January 1, 2010 to December 31, 2011. (a) Channel 1 (22.234 GHz); (b) Channel 7 (28.000 GHz); (c) Channel 13 (53.336 GHz); (d) Channel 17 (55.500 GHz).

position on the abscissa series index in Figure 2 and another at the 520th position in the abscissa series index in Figure 2 as a result of the transfer of the instrument from Shunyi $\left(40^{\circ} 07^{\prime} \mathrm{N}, 116^{\circ} 36^{\prime} \mathrm{E}, 34.0 \mathrm{~m}\right.$ a.s.l.) to Shangdianzi $\left(40^{\circ} 39^{\prime} \mathrm{N}\right.$, $117^{\circ} 07^{\prime} \mathrm{E}, 293.3 \mathrm{~m}$ a.s.l.). The value of $T_{B M}$ after liquid nitrogen calibration differs considerably from that before calibration for channels 1 and 7 , whereas the value of $T_{B M}$ for channel 13 showed a clear decrease after the instrument had been moved. The whole sample was therefore sliced into three sub-samples for the correction of $T_{B M}$.

Based on Equation (11), the coefficients $a, b$ and $c$ were derived from each of the three sub-samples for each of the 22 channels (data not shown) and Equation (9) was applied for correction. Table 1 gives the statistics for the difference between the measured and calculated brightness temperatures and the correlation coefficients before and after correction. The bias before correction was decreased to 0 after correction and the standard deviations all decreased significantly, especially for the channels in the $K$ band. The correlation coefficients between the calculated and measured brightness temperatures after correction were greater than those before correction for all 22 channels, particularly for channels 7 and 8 (as indicated by the bold font in Table 1). The correlation between $T_{B M}$ and $T_{B C}$ was almost negligible, whereas the correlation between $T_{B O}$ and $T_{B C}$ increased to about 0.9 , mainly due to the correction for the surrounding environment. The improvement in consistency is also shown by the time series for $T_{B O}$ and $T_{B C}$ shown in Figure 3 for the same four typical channels, illustrating that this method can be used to improve historical datasets. 


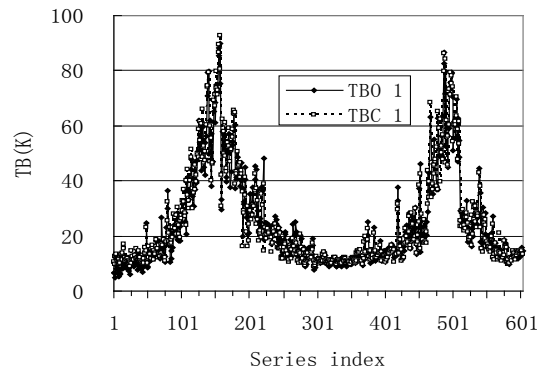

(a)

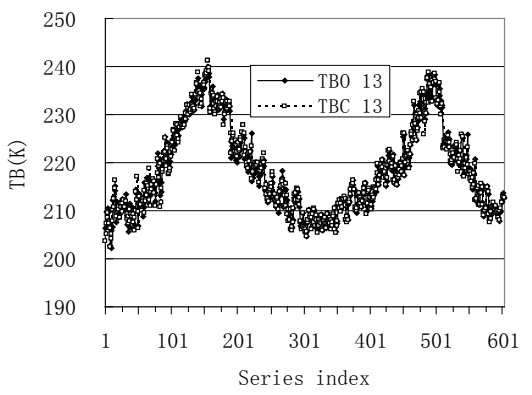

(c)

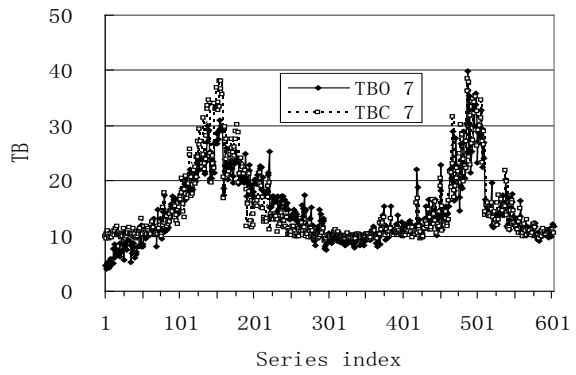

(b)

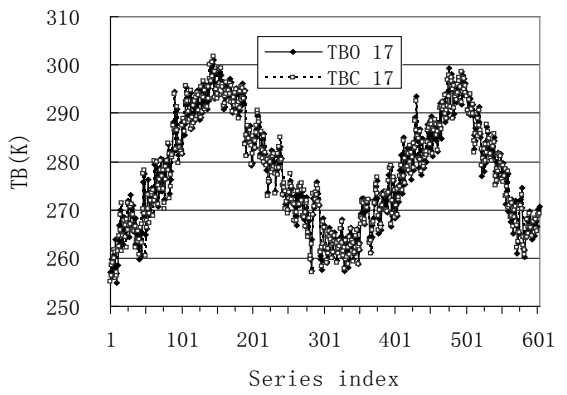

(d)

Figure 3. Comparison of the time series of the corrected observed clear sky brightness temperatures $\left(T_{B O}\right)$ with the simulated temperatures $\left(T_{B C}\right)$ for four typical channels during the period January 1, 2010 to December 31, 2011. (a) Channel 1; (b) Channel 7; (c) Channel 13; (d) Channel 17.

2) Verification with temperature and humidity profile retrievals

To verify the corrections to the brightness temperature data, we used the least-squares method to retrieve atmospheric temperature and moisture profiles from $T_{B O}$ and $T_{B M}$ respectively, and then compared the statistics of the errors in the retrieved data.

If vector $f$ is the temperature and the water vapor density profiles at $L$ height levels

$$
\boldsymbol{f}=\left[T_{1}, T_{2}, \cdots, T_{L}\right]^{\mathrm{T}}
$$

and vector $\boldsymbol{g}$ is the brightness temperatures at $K$ microwave frequencies

$$
\boldsymbol{g}=\left[T_{B 1}, T_{B 2}, \cdots, T_{B K}\right]^{\mathrm{T}}
$$

then the regression equation is

$$
\boldsymbol{f}=C * \boldsymbol{g}
$$

where $C$ is the regression coefficient matrix, and can be obtained through regression analysis by using an a regression sample (of sample size $N$ ) of both $f$ and $\boldsymbol{g}$ based on the least-squares method to give

$$
Q=\sum_{i=1}^{N} e_{i}^{2}=\sum_{i=1}^{N}(\boldsymbol{f}-C * \boldsymbol{g})^{2}=\min
$$

where $f$ is the temperature and water vapor density provided by the NCEP-FNL data and $\boldsymbol{g}$ is $T_{B C}$ plus a Gaussian random number according to $n(0, \sigma)$ to simulate the error in the brightness temperature computations, where $\sigma$ is the standard deviation given for each channel in Table 1. 
During the retrieval test with independent samples, $T_{B O}$ and $T_{B M}$ were used to replace $\boldsymbol{g}$ in Equation (14) and $\boldsymbol{f}$ obtained from Equation (14), respectively. The first 541 of the 603 samples were used as the regression samples and the last 63 samples were used as the test samples.

The 63 retrievals from $T_{B O}$ and $T_{B M}$ were compared with the NCEP-FNL data and the error statistics are shown in Figure 4(a) for temperature and in Figure 4(b) for the concentration of water vapor. Figure 4 shows that the bias from $T_{B O}$ is close to zero and the root-mean-square error (RMSE) is $<2^{\circ} \mathrm{C}$ for temperature and $1.2 \mathrm{~g} / \mathrm{m}^{3}$ for humidity up to $5 \mathrm{~km}$ height. The bias from $T_{B O}$ is better than that from $T_{B M}$. This implies that the brightness temperature after correction $\left(T_{B O}\right)$ gives better retrievals than the brightness temperature without correction $\left(T_{B M}\right)$.

The radiometer output profiles (LV2) were also compared with the NCEP-FNL data and the bias and RMSE are shown in Figure 4. The bias and RMSE for LV2 are similar to those obtained from and, in some instances, even poorer than those obtained from $T_{B M}$.

\section{Conclusions and Discussion}

This work aimed to better preserve the historical data obtained by ground-based microwave radiometers and to improve their value in future applications. We took data from the NCEP-FNL dataset as a reference and then used the radiation transfer model for forward calculation to obtain the brightness temperature. However, we found that long time series of observational data from ground-based microwave radiometers may contain segmented features (the so-called "jumping problem"). Eliminating this "jumping problem" in the brightness temperature observation data will improve both the time continuity of the brightness temperature observational data and the consistency of the system with the forward modeled data based on the NCEP-FNL dataset, allowing the observational data to be used for inversion and assimilation at a later date. Our main conclusions are as follows.

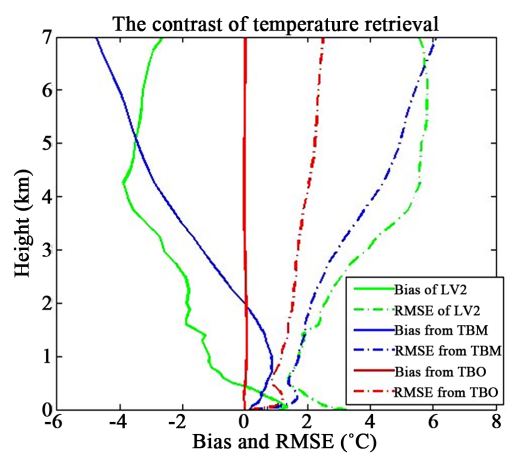

(a)

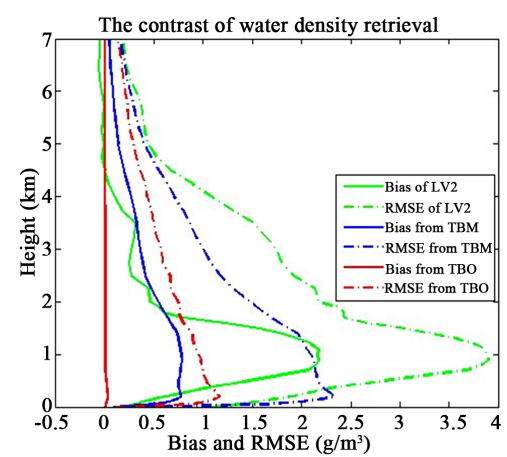

(b)

Figure 4. Comparison of error statistics for (a) temperature and (b) concentration of water vapor. Solid lines show the bias referring to the retrievals from the NCEP-FNL data; dotted lines show the root-mean-square error; green lines show the error statistics for LV2; blue lines show the error statistics for the retrievals from $T_{B M}$; and red lines show the error statistics of the retrievals from $T_{B O}$. 
1) The temperature variation around the antenna must be taken into account in the corrections, especially in the $K$ band because the spillover contributions from the side lobes and polluted radome cannot be neglected. Although frequent liquid nitrogen and tipping calibrations of the radiometer system help to alleviate the interference from the surrounding environment in the brightness temperature measurements, it is usually still necessary to correct the observations for this type of interference.

2) Our verification of the two years of observed data shows that our correction scheme is efficient. The consistency between the observations of the atmospheric brightness temperature and the ground-based microwave radiometer and forward calculations was greatly improved and the standard deviation of the difference was significantly decreased in all the channels in both the $K(20-30$ $\mathrm{GHz})$ and $V(50-60 \mathrm{GHz})$ bands, especially for channels close to $28-30 \mathrm{GHz}$.

3) We verified that the brightness temperature data after correction result in better retrievals of the atmospheric temperature and humidity than the brightness temperature data without correction.

Although our comprehensive correction procedure has significantly improved the accuracy of the calculations, this does not mean that the liquid nitrogen and tipping calibrations of the instrument systems are no longer necessary. In areas with high levels of pollution, correct calibration and maintenance of the radiometer can mitigate the degradation in the accuracy of the radiometer and regular cleaning and replacement of the radome are recommended to ensure the optimum accuracy of the observations. If the correlation and offset between the atmospheric brightness temperature observations and the ground-based microwave radiometer and forward calculations are far from the expected values, the radiometer should be suspected of not performing within its specification and should be returned to the factory.

\section{Acknowledgements}

The work is jointly supported by the National Natural Science Foundation of China (41675028, 41675029, 41275043, 41005005), The National Basic Research Program (973) of China (2013CB430102), The Project of State Key Laboratory of Severe Weather of Chinese Academy of Meteorological Sciences (2016LASW-B12), and Open Research Funding Program of KLGIS (KLGIS2015A01), Urban Meteorological Research Foundation IUMKY\&UMRF201101 and Program for Postgraduates Research Innovation of Jiangsu Higher Education Institutions (KYLX16_0948). CMA/Institute of Urban Meteorological Research in Beijing provided the observed data twice a day (0800 and 2000BT) during the period from 1 January 2010 to 31 December 2011. Drs. Li Ju, Liu Hongyan, Ruan Shunxian, Cao Xiaoyan and Mr. Shen Yonghai of this institute help a lot during data processing. NCEP FNL data is obtained from website http://rda.ucar.edu/datasets/ds083.2/ (DOI: 10.5065/D6M043C6). 


\section{Conflicts of Interest}

The authors declare no conflicts of interest regarding the publication of this paper.

\section{References}

[1] Westwater, E., Crewell, S. and Matzler, C. (2004) A Review of Surface-Based Microwave and Millimeter-Wave Radiometric Remote Sensing of the Troposphere. Radio Science Bulletin, 77, 59-80.

[2] Cimini, D., Westwater, E.R., Gasiewski, A.J., Klein, M., Leuski, V.Y. and Liljegren, J.C. (2007) Ground-Based Millimeter- and Submillimeter-Wave Observations of Low Vapor and Liquid Water Contents. IEEE Transactions on Geoscience and Remote Sensing, 45, 2169-2180. https://doi.org/10.1109/TGRS.2007.897450

[3] Cimini, D., Campos, E., Ware, R., Albers, S., Giuliani, G., Oreamuno, J., Joe, P., Koch, S.E., Cober, S. and Westwater, E. (2011) Thermodynamic Atmospheric Profiling during the 2010 Winter Olympics Using Ground-Based Microwave Radiometry. IEEE Transactions on Geoscience and Remote Sensing, 49, 4959-4969. https://doi.org/10.1109/TGRS.2011.2154337

[4] Cimini, D., Nelson, M., Güldner, J. and Ware, R. (2015) Forecast Indices from Ground-Based Microwave Radiometer for Operational Meteorology. Atmospheric Measurement Techniques, 8, 315-333. https://doi.org/10.5194/amt-8-315-2015

[5] Güldner, J. and Spänkuch, D. (2001) Remote Sensing of the Thermodynamic State of the Atmospheric Boundary Layer by Ground-Based Microwave Radiometry. Journal of Atmospheric and Oceanic Technology, 18, 925-933. https://doi.org/10.1175/1520-0426(2001)018<0925:RSOTTS >2.0.CO;2

[6] Hewison, T. (2007) 1D-VAR Retrievals of Temperature and Humidity Profiles from a Ground-Based Microwave Radiometer. IEEE Transactions on Geoscience and Remote Sensing, 45, 2163-2168. https://doi.org/10.1109/TGRS.2007.898091

[7] Maschwitz, G., Löhnert, U., Crewell, S., Rose, T. and Turner, D.D. (2013) Investigation of Ground-Based Microwave Radiometer Calibration Techniques at $530 \mathrm{hPa}$. Atmospheric Measurement Techniques, 6, 2641-2658. https://doi.org/10.5194/amt-6-2641-2013

[8] Stähli, O., Murk, A., Kämpfer, N., Mätzler, C. and Eriksson, P. (2013) Microwave Radiometer to Retrieve Temperature Profiles from the Surface to the Stratopause. Atmospheric Measurement Techniques, 6, 2477-2494. https://doi.org/10.5194/amt-6-2477-2013

[9] Illingworth, A., Cimini, D., Gaffard, C., Haeffelin, M., Lehmann, V., Löhnert, U., O’Connor, E. and Ruffieux, D. (2015) Exploiting Existing Ground-Based Remote Sensing Networks to Improve High-Resolution Weather Forecasts.

[10] Ware, R., Solheim, F., Carpenter, R., Güldner, J., Liljegren, J., Nehrkorn, T. and Vandenberghe, F. (2003) A Multi-Channel Radiometric Profiler of Temperature, Humidity and Cloud Liquid. Radiologic Science, 38, 8079-8032.

[11] Ware, R., Cimini, D., Campos, E., Giuliani, G., Albers, S., Nelson, M., Koch, S. E., Joe, P. and Cober, S. (2013) Thermodynamic and Liquid Profiling during the 2010 Winter Olympics. Atmospheric Research, 132, 278-290. https://doi.org/10.1016/j.atmosres.2013.05.019

[12] Campos, E.F., Ware, R., Joe, P. and Hudak, D. (2014) Monitoring Water Phase Dynamics in Winter Clouds. Atmospheric Research, 147-148, 86-100. https://doi.org/10.1016/j.atmosres.2014.03.008

[13] Serke, D., Hall, E., Bognar, J., Jordan, A., Abdo, S., Baker, K., Seitel, T., Nelson, M., 
Reehorst, A., Ware, R., McDonough, F. and Politovich, M. (2014) Supercooled Liquid Water Content Profiling Case Studies with a New Vibrating Wire Sonde Compared to a Ground-Based Microwave Radiometer. Atmospheric Research, 149, 77-87. https://doi.org/10.1016/j.atmosres.2014.05.026

[14] Wang, Z.H., Li, Q., Hu, F.C., Cao, X.F. and Chu, Y.L. (2014) Remote Sensing of Lightning by a Ground-Based Microwave Radiometer. Atmospheric Research, 150, 143-150. https://doi.org/10.1016/j.atmosres.2014.07.009

[15] Huang, J.P., He, M., Yan, H.R., et al. (2010) A Study of Liquid Water Path and Precipitable Water Vapor in Lanzhou Area Using Ground-Based Microwave Radiometer. Chinese Journal of Atmospheric Sciences, 34, 548-558.

[16] Liu, H.Y. (2011) The Temperature Profile Comparison between the Ground-Based Microwave Radiometer and the Other Instrument for the Recent Three Years. Acta Meteorologica Sinica, 69, 719-728.

[17] Li, F., Wu, D., Tan, H.B., Bi, X.Y., Jiang, D.H., Deng, T., Chen, H.H. and Deng, X.J. (2012) The Characteristics and Causes Analysis of a Typical Haze Process during the Dry Season over Guangzhou Area: A Case Study. Journal of Tropical Meteorology, 28, 113-122.

[18] Guo, L.J. and Guo, X.L. (2015) Verification Study of the Atmospheric Temperature and Humidity Profiles Retrieved from the Ground-Based Multi-Channels Microwave Radiometer for Persistent Foggy Weather Events in Northern China. Acta Meteorologica Sinica, 73, 368-381.

[19] Xu, G., Ware, R., Zhang, W., Feng, G., Liao, K. and Liu, Y. (2014) Effect of Off-Zenith Observation on Reducing the Impact of Precipitation on Ground-Based Microwave Radiometer Measurement Accuracy in Wuhan. Atmospheric Research, 140-141, 85-94. https://doi.org/10.1016/j.atmosres.2014.01.021

[20] Bao, Y.S., Qian, C., Min, J.Z., Hou, Y.Y. and Lu, Q.F. (2016) 0-10 km Temperature and Humidity Profiles Retrieval from Ground-Based Microwave Radiometer. Journal of Tropical Meteorology, 32, 163-171.

[21] Westwater, E., Wang, Z.H., Grody, N.C. and McMillin, L.M. (1985) Remote Sensing of Temperature Profiles from a Combination of Observations from the Satellite-Based Microwave Sounding Unit and the Ground-Based Profiler. Journal of Atmospheric and Oceanic Technology, 2, 97-109. https://doi.org/10.1175/1520-0426(1985)002<0097:RSOTPF >2.0.CO;2

[22] Solheim, F., Godwin, J., Westwater, E., Han, Y., Keihm, S., Marsh, K. and Ware, R. (1998) Radiometric Profiling of Temperature, Water Vapor, and Cloud Liquid Water Using Various Inversion Methods. Radio Science, 33, 393-404. https://doi.org/10.1029/97RS03656

[23] Lu, Q., Bell, W., Bauer, P., Bormann, N., et al. (2010) An Initial Evaluation of FY-3A Satellite Data. ECMWF Technical Memorandum 631, European Centre for Medium-Range Weather Forecasts, 58.

[24] Goldberg, M.D., Crosby, D.S. and Zhou, L. (2001) The Limb Adjustment of Amsua Observations: Methodology and Validation. Journal of Applied Meteorology and Climatology, 40, 70-83. https://doi.org/10.1175/1520-0450(2001)040<0070:TLAOAA >2.0.CO;2

[25] Weng, F.Z., Zhao, L., Ferraro, R.R., Poe, G., Li, X. and Grody, N.C. (2003) Advanced Microwave Sounding Unit Cloud and Precipitation Algorithms. Radio Science, 38, 8068-8096. https://doi.org/10.1029/2002RS002679

[26] Weng, F.Z., Yang, H. and Zou, X.L. (2013) On Convertibility from Antenna to Sensor Brightness Temperature for ATMS. IEEE Geoscience and Remote Sensing Letters, 10, 771-775. https://doi.org/10.1109/LGRS.2012.2223193 
[27] Han, Y. and Westwater, E.R. (2000) Analysis and Improvement of Tipping Calibration for Ground-Based Microwave Radiometers. IEEE Transactions on Geoscience and Remote Sensing, 38, 1260-1276. https://doi.org/10.1109/36.843018

[28] Meunier, V., Lohnert, U., Kollias, P. and Crewell, S. (2013) Biases Caused by the Instrument Bandwidth and Beam Width on Simulated Brightness Temperature Measurements from Scanning Microwave Radiometers. Atmospheric Measurement Techniques, 6, 1171-1187. https://doi.org/10.5194/amt-6-1171-2013

[29] D’orazio, A., DeSario, M., Gramegna, T., et al. (2003) Optimisation of Tipping Curve Calibration of Microwave Radiometer. Electronics Letters, 39, 905-906. https://doi.org/10.1049/el:20030622

[30] Li, J.M., Guo, L.X., Lin, L.K., Zhao, Y.Y. and Cheng, X.H. (2014) A New Method of Tipping Calibration for Ground-Based Microwave Radiometer in Cloudy Atmosphere. IEEE Transactions on Geoscience and Remote Sensing, 52, 5506-5513. https://doi.org/10.1109/TGRS.2013.2290013

[31] Wang, Z.H., Cao, X.F., Huang, J.S., et al. (2014) Analysis on the Working State of a Ground Based Microwave Radiometer Based on Radiative Transfer Model and Meteorological Data Variation Features. Transactions of Atmospheric Sciences, 37, 1-8.

[32] Li, Q., Hu, F.C., Chu, Y.L., Wang, Z.H., Huang, J.S., Wang, Y. and Zhu, Y.Y. (2014) A Consistency Analysis and Correction of the Brightness Temperature Data Observed with a Ground Based Microwave Radiometer in Beijing. Remote Sensing Technology and Application, 29, 547-556.

[33] National Centers for Environmental Prediction/National Weather Service/NOAA/U.S. Department of Commerce (2000) NCEP FNL Operational Model Global Tropospheric Analyses, Continuing from July 1999. Research Data Archive at the National Center for Atmospheric Research; Computational and Information Systems Laboratory, Boulder.

[34] Decker, M.T., Westwater, E.R. and Guiraud, F.O. (1978) Experimental Evaluation of Ground-Based Microwave Radiometric Sensing of Atmospheric Temperature and Water Vapor Profiles. Journal of Applied Meteorology and Climatology, 17, 1788-1795. https://doi.org/10.1175/1520-0450(1978)017<1788:EEOGBM>2.0.CO;2

[35] Liebe, H.J., Rosenkranz, P.W. and Hufford, G.A. (1992) Atmospheric 60 GHz Oxygen Spectrum: New Laboratory Measurements and Line Parameters. Journal of Quantitative Spectroscopy \& Radiative Transfer, 48, 629-643. https://doi.org/10.1016/0022-4073(92)90127-P

[36] Liebe, H.J. (1985) An Updated Model for Millimeter Wave Propagation in Moist Air. Radio Science, 20, 1069-1089. https://doi.org/10.1029/RS020i005p01069

[37] Ao, X., Wang, Z.H., Xu, G.R., et al. (2013) Study on the Quality Control of Brightness Temperature Data Observed with Ground-Based Microwave Radiometer. Scientia Meteorologica Sinica, 33, 130-137.

[38] Wang, Y., Wang, Z.H., Li, Q. and Zhu, Y.Y. (2014) Research of the One-Dimensional Variational Algorithm for Retrieving Temperature and Humidity Profiles from the Ground-Based Microwave Radiometer. Acta Meteorologica Sinica, 72, 570-582.

[39] Zhu, Y.Y., Wang, Z.H., Chu, Y.L., Wang, Y. and Li, Q. (2015) Comprehensive Quality Control on Brightness Temperature Data Observed with a Ground-Based Microwave Radiometer and the Efficiency Analysis. Remote Sensing Technology \& Application, 35, 621-628.

[40] Ulaby, F.T., Moore, R.K. and Fung, A.K. (1981) Microwave Remote Sensing: Active and Passive. Vol. 1 Microwave Remote Sensing Fundamentals and Radiometry. Addison-Wesley Publishing Company, New York, 2162. 